003.3 THE EUROPEAN GONOCOCCAL ANTIMICROBIAL SURVEILLANCE PROGRAMME FINDINGS 2017

${ }^{1}$ Michaela Day*, 'Michelle Cole, ${ }^{2}$ Gianfranco Spiteri, ${ }^{3}$ Susanne Jacobsson, ${ }^{1}$ Neil Woodford, ${ }^{2}$ Andrew Amato-Gauci, ${ }^{3}$ Magnus Unemo. ${ }^{1}$ Public Health England, National Infection Service, London, UK; ${ }^{2} E C D C$, Sweden; ${ }^{3}$ Örebro University Hospital, ÖREBRO, Sweden

10.1136/sextrans-2019-sti.118

Background The European Gonococcal Antimicrobial Surveillance Programme (Euro-GASP) annually investigates antimicrobial susceptibility data for Neisseria gonorrhoeae with patient epidemiological data to monitor current and emerging trends in antimicrobial resistance (AMR) across Europe. Susceptibility to ceftriaxone and azithromycin, currently recommended for combination treatment in the European management guideline, has decreased in the past; regular surveillance of AMR is crucial. We present the main Euro-GASP findings from 2017.

Methods Agar dilution and minimum inhibitory concentration (MIC) gradient strip tests were used to determine the antimicrobial susceptibility to cefixime, ceftriaxone and azithromycin (using EUCAST breakpoints) of $3248 \mathrm{~N}$. gonorrhoeae isolates collected in 2017 from 27 countries across the European Union/ European Economic Area (EU/EEA). Significance of changes in resistance compared to 2016 was analysed using Z-tests.

Results There were no isolates with ceftriaxone resistance $(\mathrm{MIC}>0.125 \mathrm{mg} / \mathrm{L}$ ) (zero in 2016), 7.5\% of isolates were azithromycin resistant $(\mathrm{MIC}>0.5 \mathrm{mg} / \mathrm{L})(7.5 \%$ in $2016 ; \mathrm{p}=0.93)$ and cefixime resistance (MIC>0.125 mg/L) was observed in $1.9 \%$ of isolates $(2.1 \%$ in $2016 ; \mathrm{p}=0.53)$. Seven isolates from four countries displayed high-level azithromycin resistance $(\mathrm{MIC} \geq 256 \mathrm{mg} / \mathrm{L})$, which is the same number as observed in 2016, although in different countries (five countries in 2016). Ceftriaxone MICs for 28 isolates (0.9\%) were $0.125 \mathrm{mg} / \mathrm{L}$ (on the resistance breakpoint) which is double the number observed in 2016 (14 isolates, 0.5\%) although this increase is not statistically significant $(p=0.33)$. Of the 28 isolates on the ceftriaxone resistance breakpoint, four showed intermediate susceptibility to azithromycin.

Conclusion Ceftriaxone, azithromycin and cefixime resistance levels remained stable compared with 2016. However, the current azithromycin resistance rate of $7.5 \%$ and the number of isolates on the resistance breakpoint for ceftriaxone threaten the effectiveness of the currently recommended European therapeutic regimen of ceftriaxone $500 \mathrm{mg}$ plus azithromycin $2 \mathrm{~g}$. Continued surveillance is essential together with, ultimately, development of new effective antimicrobials.

Disclosure No significant relationships.

\section{O03.4 MODELLING INTERVENTION STRATEGIES FOR PREVENTING SPREAD OF EXTENSIVELY DRUG RESISTANT GONORRHOEA STRAINS AMONG AUSTRALIAN MSM}

'Qibin Duan*, 'James Wood, 'Ben Hui, 'David Regan. 'UNSW Sydney, The Kirby Institute, Sydney, Australia; ${ }^{2}$ UNSW Sydney, School of Public Health and Community Medicine, Sydney, Australia

\subsection{6/sextrans-2019-sti.119}

Background Recent reports from Australia and the UK of extensively drug resistant (XDR) gonorrhoea strains, have increased concerns over remaining treatment options. Outbreak-type responses to detection of such strains may help to delay wider emergence of such resistance but lack a clear evidence base as to their effect. Here, we use mathematical models to assess the potential impact of outbreak response strategies in a high-incidence population of Australian men who have sex with men (MSM) with gonorrhoea.

Methods We developed an individual-based, anatomical sitespecific model of gonorrhoea transmission in Australian MSM. The model was calibrated to available site-specific prevalence and incidence data with respect to the per-act transmission probabilities for four types of sexual practice. As pharyngeal importations were most difficult to control, we focused on these in estimating the probability of elimination of an imported XDR strain. We considered various combinations of contact tracing and screening interventions, with results for each scenario based on 5000 simulations.

Results At current levels of gonorrhoea screening in Australian MSM, we predict persistence of secondary spread at 5 years post-importation in just under $20 \%$ of simulations. If all infected regular partners of index patients are traced and treated, this persistence probability declines to $\sim 8 \%$, and further to $\sim 4 \%$ and $0.04 \%$, respectively, if $20 \%$ and $50 \%$ of all casual partners in the last two months are traced and treated. Alternatively, If the screening rate is increased to the level recommended in STI management guidelines, the probability of persistence after 5 years is $\sim 9.7 \%$. When combined with treatment of regular partners, this probability is reduced to $<0.01 \%$.

Conclusion This study suggests that contact tracing and screening rate can separately play an important role in responding to outbreaks of XDR gonorrhoea, and in combination these strategies may have the potential to prevent domestic establishment of such strains.

Disclosure No significant relationships.

\subsection{UTILITY OF REAL-TIME WHOLE GENOME SEQUENCING IN PARTNER NOTIFICATION AND CONTROL OF NEISSERIA GONORRHOEAE INFECTION}

${ }^{1}$ Ling Yuan Kong*, 'Ines Moura, 'Warren Fawley, ${ }^{2}$ Janet Wilson, ${ }^{2}$ Laura Kelly, ${ }^{3} \mathrm{~A}$ Sarah Walker, ${ }^{4}$ David Eyre, ${ }^{1}$ Mark Wilcox. 'Leeds Teaching Hospitals NHS Trust, Microbiology, Leeds, UK; ${ }^{2}$ Leeds Teaching Hospitals NHS Trust, Leeds Sexual Health, Leeds, UK; ${ }^{3}$ Oxford University, Nuffield Department Of Medicine, Oxford, UK; ${ }^{4}$ University of Oxford, Nuffield Department Of Medicine, Oxford, UK

\subsection{6/sextrans-2019-sti.120}

Background Gonorrhoea is a sexually transmitted infection of global public health concern. We investigated whole genome sequencing (WGS) as a partner notification (PN) tool in gonorrhoea management.

Methods Between May-November 2018, all N. gonorrhoeae isolated from patients attending Leeds Sexual Health, UK, underwent WGS. Sequences were compared with historical isolates from Leeds, 2016 onwards. Reports listing sequences within 20 single nucleotide polymorphisms (SNPs) were issued to clinicians. Patient and PN data were reviewed; numbers of traceable and untraceable partners were determined. Reports were reviewed to confirm WGS links between traceable partners and to identify possible links for untraceable partners, as determined by a transmission nomogram and epidemiological match (gender, sexual orientation, onset of symptoms, and other identifiers e.g. name). Clusters of cases within 20 SNPs were examined for patterns.

Results Overall 380 isolates from 377 cases were successfully sequenced. Traceable partners were found in 244 cases. 147 